\begin{tabular}{|c|c|c|}
\hline Beitr. Ent. & Keltern & ISSN 0005-805X \\
\hline $\mathbf{6 0}(2010) 2$ & S. $259-280$ & 20.12 .2010 \\
\hline
\end{tabular}

\title{
Biodiversità delle Aleocharinae della Cina: Placusini, Homalotini, Bolitocharini, Eusteniamorphini e Falagriini
}

\section{(Coleoptera, Staphylinidae)}

Con 45 figure

\section{Roberto Pace}

\section{Summary}

In the present paper 33 species are listed, 14 of which are described and illustrated as new for the Science, one of the genus Placusa (P. shimianensis n. sp.), three of the genus Coenonica (C. guanxiensis n. sp., C. confundibilis n. sp., C. anteopaca n. sp.), one of the genus Stenomastax (S. chinensis n. sp.), one of the new genus Tibetusina (T. wolongensis n. sp.), two of the genus Pseudatheta (P. similis n. sp., P. chinensis n. sp.), one of the genus Phymatura (P. sinica n. sp.), one of the genus Bolitochara (B. sinica n. sp.), one of the genus Eusteniamorpha (E. jiajinensis n. sp.), and three of the genus Falagria (F. shimianensis n. sp., F. hubeiensis n. sp., F. magnifica n. sp.).

Key words

Coleoptera, Staphylinidae, Aleocharinae, taxonomy, China

\section{Zusammenfassung}

In der vorliegenden Arbeit werden 33 Arten aufgelistet. Davon werden 14 Arten sowie eine Gattung als neu für die Wissenschaft beschrieben und abgebildet: eine Art der Gattung Placusa (P. shimianensis n. sp.), drei der Gattung Coenonica (C. guanxiensis n. sp., C. confundibilis n. sp., C. anteopaca n. sp.), eine der Gattung Stenomastax (S. chinensis n. sp.), eine der neuen Gattung Tibetusina n. gen. (T. wolongensis $\mathrm{n}$. sp.), zwei der Gattung Pseudatheta (P. similis n. sp., P. chinensis n. sp.), eine der Gattung Phymatura (P. sinica n. sp.), eine der Gattung Bolitochara (B. sinica n. sp.), eine der Gattung Eusteniamorpha (E. jiajinensis n. sp.) und drei der Gattung Falagria (F. shimianensis n. sp., F. hubeiensis n. sp., F. magnifica n. sp.).

\section{Riassunto}

Nel presente lavoro sono elencate 33 specie, 14 delle quali sono descritte e illustrate come nuove per la Scienza, una del genere Placusa (P. shimianensis n. sp.), tre del genere Coenonica (C. guanxiensis n. sp., C. confundibilis n. sp., C. anteopaca n. sp.), una del genere Stenomastax (S. chinensis n. sp.), una del nuovo genere Tibetusina (T. wolongensis n. sp.), due del genere Pseudatheta (P. similis n. sp., P. chinensis n. sp.), una del genere Phymatura (P. sinica n. sp.), una del genere Bolitochara (B. sinica n. sp.), una del genere Eusteniamorpha (E. jiajinensis n. sp.) e tre del genere Falagria (F. shimianensis n. sp., F. hubeiensis n. sp., F. magnifica n. sp.).

\footnotetext{
${ }^{1} 244^{\circ}$ Contributo alla conoscenza delle Aleocharinae.
} 


\section{Introduzione}

In questi ultimi anni le ricerche entomologiche si sono particolarmente intensificate in Cina, con conseguente incremento di pubblicazioni riguardanti lo studio tassonomico di Aleocharinae. In questo ambito si inserisce anche il presente lavoro che è un contributo a una sempre migliore conoscenza sulla tassonomia di questa sottofamiglia degli Staphylinidae della Cina.

\section{Materiale e metodo}

Gli esemplari del presente lavoro sono stati raccolti recentemente (1997, 1999, 2001, 2003) dai colleghi studiosi di Staphylinidae Dr. Aleš Smetana di Ottawa e Michael Schülke di Berlino. Comprende anche le raccolte di Andreas Pütz a me affidate in studio dal Dr. Olaf Jäger del Senckenberg Museum für Tierkunde di Dresda.

Anche lo studio tassonomico delle specie cinesi della sottofamiglia Aleocharinae, come quello d'altre regioni zoogeografiche, presenta seri problemi interpretativi che sono risolti in gran parte grazie all'esame della forma dell'organo copulatore maschile (edeago) e della spermateca. Gli esemplari sono stati dissezionati con lo scopo di includere le strutture genitali in balsamo del Canadà (su piccoli rettangoli trasparenti di materiale di plastica, che accompagnano gli esemplari). Le strutture genitali sono state studiate con l'uso di un microscopio composto fino a 450 ingrandimenti e disegnate per mezzo di oculare a reticolo. Le misurazioni sono state eseguite mediante l'ausilio di un oculare con scala micrometrica. I caratteri dell'habitus sono stati osservati con microscopio stereoscopico fino a 100x. Tutti i disegni delle tavole e le foto sono stati da me eseguiti fino alla fase finale. Le tavole sono state da me composte al computer.

\section{Acronimi}

Gli holotypi e paratypi delle nuove specie sono conservati nei seguenti istituti:

$\begin{array}{ll}\text { SDEI } & \text { Senckenberg Deutsches Entomologisches Institut di Müncheberg } \\ \text { SMTD } & \text { Senckenberg Museum für Tierkunde, Dresden } \\ \text { MNHU } & \text { Museum für Naturkunde Humboldt Universität, Berlin } \\ \text { IRSNB } & \text { Institut royal des Sciences naturelles de Belgique, Bruxelles }\end{array}$

Altri paratypi in collezione Smetana.

\section{Placusini Mulsant \& Rey, 1871}

\section{Placusa Erichson, 1837}

\section{Placusa shimianensis n. sp.}

(Figg. 1 e 15-16)

\section{Materiale tipico:}

Holotypus ơ, China: W-Sichuan, Ya’an Prefecture, Shimian Co., Xiaoxiang Ling, side valley ab. Nanya Cun, nr. Caluo, 11 km S Shimian, 1250 m, 7.VII.1999, leg. A. Pütz (SMTD).

Paratypus: $1 \sigma^{\star}$, stessa provenienza (SMTD). 


\title{
Descrizione:
}

Lunghezza 2,8 $\mathrm{mm}$. Corpo debolmente lucido e giallo-bruno, capo bruno, antenne brune con base dei due antennomeri basali gialla, zampe giallo-brune. Secondo antennomero più corto del primo, terzo lungo quanto il secondo, antennomeri quarto a decimo trasversi. Reticolazione di capo e pronoto assente, quella delle elitre evidente, quella dell'addome forte, Granulosità dell'avancorpo fittissima e saliente, quella dell'addome superficiale. Edeago fig. 15, sesto urotergo libero del ơ fig. 16.

\section{Comparazioni:}

Per la forma dell'edeago e del sesto urotergo libero del $\sigma^{*}$ la nuova specie è simile a $P$. spinigera KraATZ, 1859 dello Sri Lanka, di cui ho esaminato la serie tipica di $80^{\star} o^{\star}$ e 2 ㅇ ㅇ (SDEI). La nuova specie se ne distingue per l'edeago tozzo, con lama sternale ricurva e larga (edeago slanciato e lama sternale stretta e quasi rettilinea in spinigera. Le spine laterali del sesto urotergo libero del $\sigma^{t}$ sono lunghe quasi quanto i lobi interni, mentre in spinigera le spine laterali del sesto urotergo libero del $\sigma^{\top}$ sono assai lunghe, nettamente più lunghe dei lobi interni.

\section{Etimologia:}

La nuova specie prende nome dalla regione dello Shimian, dove è stata raccolta.

\section{Homalotini Heer, 1839}

\author{
Neosilusa Cameron, 1920
}

\section{Neosilusa ceylonica (KrAATZ, 1857)}

Stenusa ceylonica Kratz, 1857: 8

Plagiusa ceylonica: Cameron, 1939: 167

Neosilusa ceylonica: PACE 1984: 15; PACE 1993: 71

7 es., China, Sichuan, Qingcheng Shan, 65 km NW Chengdu, 3053'N 1033', 800-1000 m, 18.V/3-4.VI.1999 m, 8 km W Taiping.VI.1997, leg. A. Pütz; 22 es., China: W-Sichuan, Ganzi Tibet. Aut. Prefecture, Luding Co., 7 km S Luding, tributary of Dadu He, $29^{\circ} 53.15^{\prime} \mathrm{N}$ 102¹2.78'E, 23.VI.1999, leg. A. Pütz; 1 es., China: W-Sichuan, Ya’an Prefecture, Shimian Co., Daxue Shan, W road btw. Anshunchang-Wanba, 20 km WSW Shimian, 1500 m, 9.VII.1999, leg. A. Pütz; 1 es., China: W-Sichuan, Ya’an Prefecture, Ya’an Co., road 108, ca. 20 km S Ya’an, ca. 1000 m, 19.VI.1999, leg. A. Pütz.

\section{Distribuzione:}

Specie distribuita dalle Mascarene, all'India, Malesia, Cina e Giappone.

Nota:

Esemplari comparati con quelli della serie tipica da me esaminati. 


\section{Coenonica Kraatz, 1857}

\section{Coenonica zhejiangensis PACE, 1998}

Coenonica zhejiangensis PACE, 1998: 188

$10^{\star}$, China: W-Sichuan, Yảan Prefecture, Tianquan Co., W Erlang Shan, Pass $2780 \mathrm{~m}$, 21.VI.1999, 2951'27'N $21^{\circ} 15^{\prime} 47^{\prime \prime}$, sifted, leg. A. Pütz.

\section{Distribuzione:}

Specie finora nota dello Zhejiang.

\section{Coenonica truncata PACE, 1998}

Coenonica truncata PACE, 1998: 186

$10^{\star}$, China: W-Sichuan, Yảan Prefecture, Shimian Co., Xiaoxiang Ling, side valley ab. Nanya Cun, nr. Caluo, 11 km S Shimian, 1250 m, 7.VII.1999, leg. A. Pütz.

\section{Distribuzione:}

Specie finora nota dello Yunnan.

\section{Coenonica sharpi (FAUVEL, 1901)}

Sipalia sharpi Fauvel, 1901: 66

Leptusa sharpi: Bernhauer \& SCheerpeltz, 1926: 557

Coenonica sharpi: PACE, 1989: 23

1 ㅇ, China: Sichuan, Ganzi Prefecture, Daxue Shan, Gongga Shan, Hailougou glacier park, $102^{\circ} 04^{\prime} \mathrm{E} 29^{\circ} 36^{\prime} \mathrm{N}$, river valley, ca. $1 \mathrm{~km}$ above camp I, $2100 \mathrm{~m}, 28-31 . V .1997$, leg. A. Pütz; 1 ㅇ, China: Sichuan, Gongga Shan, Hailougou glacier park, above camp II, 3000 m, 30.V.1997, sifted, leg. A. Pütz.

\section{Distribuzione:}

Specie finora nota del Giappone.

Nota:

Esemplari comparati con quelli della serie tipica da me esaminati.

\section{Coenonica ming PACE, 1993}

Coenonica ming PACE, 1993: 80

4 es., China, Sichuan, Qingcheng Shan, 65 km NW Chengdu, 3053'N 10333'E, 800-1000 m, 18.V/3-4.VI.1999 m, 8 km W Taiping.VI.1997, leg. A. Pütz.

\section{Distribuzione:}

Specie finora nota dello Yunnan e Guizhou. 
Beitr. Ent. 60 (2010) 2

Coenonica absurda PACE, 1998

Coenonica absurda PACE, 1998: 183

1 ㅇ China: W-Sichuan, Ya’an Prefecture, Shimian Co., Xiaoxiang Ling, side valley ab. Nanya Cun, nr. Caluo, 11 km S Shimian, 1250 m, 7.VII.1999, leg. A. Pütz.

Distribuzione:

Specie finora nota di Hong Kong.

\section{Coenonica guanxiensis $\mathrm{n}$. sp.}

(Figg. 2 e 17-19)

\section{Materiale tipico:}

Holotypus o, China: Guangxi, Cenwanglaoshan, 1550 m, 1.VIII.1999, leg. J. R. Fellow (IRSNB).

\section{Descrizione:}

Lunghezza $3 \mathrm{~mm}$. Capo opaco sulla metà posteriore, resto del corpo lucido. Corpo rossiccio, antenne rossicce con i due antennomeri basali giallo-rossicci, zampe giallo-rossicce. Secondo antennomero più corto del primo, terzo più corto del secondo, antennomeri quarto a settimo trasversi, antennomeri restanti perduti. Reticolazione del capo forte solo sulla metà posteriore, in avanti assente, resto del corpo senza reticolazione. Punteggiatura del capo fitta. Granulosità del pronoto saliente, quella delle elitre forte. Solco mediano frontale del capo con una fossetta davanti ad esso. Solco del pronoto a forma di U. Edeago figg. 17-18, sesto urotergo libero del ơ fig. 19.

\section{Comparazioni:}

Per la forma dell'edeago la nuova specie è simile a C. ming PACE, 1993 dello Yunnan. L'edeago della nuova specie è meno profondamente arcuato al lato ventrale e ha "crista apicalis» nettamente più stretta di quella di ming. Il solco mediano a $U$ del pronoto della nuova specie manca sul pronoto di ming.

\section{Etimologia:}

La nuova specie prende nome dalla località di raccolta.

\section{Coenonica confundibilis n. sp.}

(Figg. 3 e 20)

\section{Materiale tipico:}

Holotypus 우 , China: Border Shaanxi-Sichuan (Daba Shan), pass $20 \mathrm{~km}$ SSE Zhenping, 1700$1800,31^{\circ} 44^{\prime} \mathrm{N} 109^{\circ} 35^{\prime} \mathrm{E}, 9 . \mathrm{VII} .2001$, young dry mixed forest, field edge, small creek valley, moss, sifted, leg. M. Schülke (MNHU).

\section{Descrizione:}

Lunghezza 2,5 mm. Corpo lucido e bruno-rossiccio, capo bruno, antenne brune con i due antennomeri basali bruno-rossicci, zampe giallo-rossicce. Secondo antennomero più corto del terzo, quarto trasverso, antennomeri quinto a decimo fortemente trasversi. Reticolazione del corpo 
assente. Punteggiatura del capo fitta ed evidente, ma assente sulla fronte, quella delle elitre ben impressa. Granulosità del pronoto evidente, quella dell'addome saliente, particolarmente presso il margine posteriore di ciascun urotergo libero. Pronoto con due deboli solchi mediani sulla metà posteriore. Spermateca fig. 20.

\section{Comparazioni:}

Per la forma della spermateca e del pronoto, la nuova specie è simile a C. angularis PACE, 1998 dello Zhejiang, ma l'introflessione apicale del bulbo distale della spermateca è profondo nella nuova specie, appena sporgente in angularis e gli angoli posteriori del pronoto della nuova specie sono ampiamente ottusi, mentre in angularis sono di poco ottusi.

\section{Etimologia:}

La nuova specie avendo habitus simile a quello di specie del genere Pseudoplandria è chiamata «Confondibile» se non si esaminano i caratteri generici.

\section{Coenonica anteopaca n. sp.}

(Figg. 4 e 21-22)

\section{Materiale tipico:}

Holotypus o ${ }^{\star}$, China: N.Yunnan, Lijiang, Naxi Co., 3 km NW Yongsheng, 53 km WSW Lijiang, 2641.8'N 10043.1'E, 1990 m, 18.VIII.2003, leg. A. Smetana (SDEI).

Paratypus: 1 đ̆, Chiana: Sichuan, Baoxing, 100 km N Yaan, 12-14.VII.1995, leg. Z. Jindra.

\section{Descrizione:}

Lunghezza $2 \mathrm{~mm}$. Capo e pronoto opachi, resto del corpo lucido. Corpo nero-bruno, margini posteriori degli uroterghi liberi basali primo, secondo e terzo rossicci, antenne nere, zampe gialle. Secondo antennomero lungo quanto il primo, terzo molto più corto del secondo, antennomeri quarto a decimo fortemente trasversi. Capo e pronoto rugosi a motivo della granulosità fittissima. Reticolazione delle elitre assente, quella dell'addome trasversa e assai superficiale solo sugli uroterghi liberi quarto e quinto. Granulosità fitta alla base dell'addome, rada sul quinto urotergo libero del $o^{\top}$.

\section{Comparazioni:}

La nuova specie presenta capo e pronoto molto opachi come in C. semimutata PACE, 1998, pure dello Yunnan, ma l'edeago è poco arcuato al lato ventrale nella nuova specie, assai arcuato in semimutata. Il pronoto è solcato a U posteriormente in C. semimutata, mentre il pronoto della nuova specie è privo di solchi evidenti.

\section{Etimologia:}

La nuova specie ha nome che significa «Opaca anteriormente» a motivo dell'opacità di capo e pronoto. 
Stenomastax CAMERon, 1933

\section{Stenomastax chinensis n. sp.}

(Figg. 5 e 23-25)

\section{Materiale tipico:}

Holotypus ơ , China: W-Sichuan, Ya’an Prefecture, Shimian Co., Xiaoxiang Ling, side valley ab. Nanya Cun, nr. Caluo, 11 km S Shimian, 1250 m, 7.VII.1999, leg. A. Pütz (SMTD).

\section{Descrizione:}

Lunghezza $3 \mathrm{~mm}$. Avancorpo opaco, addome lucido. Corpo bruno, capo nero-bruno, pigidio giallo-rossiccio, antenne brune con i tre antennomeri basali rossicci, zampe giallo-rossicce. Secondo antennomero più corto del primo, terzo più corto del secondo, antennomeri quarto e quinto lunghi quanto larghi, sesto a decimo trasversi. Occhi lunghi quanto le tempie. Reticolazione delle elitre evidente, quella dell'addome poligonale irregolare netta. Punteggiatura di capo e pronoto fittissima, tanto da dare un aspetto opaco alla superficie. Granulosità dell'addome poco fitta e superficiale. Edeago figg. 24-25.

\section{Comparazioni:}

Per la forma dell'edeago la nuova specie si pone sistematicamente vicino a $S$. tuberculicollis (KraAtz, 1859) dello Sri Lanka, Borneo e Giava, di cui ho esaminato la serie tipica di 3 o + e $\sigma^{\star} o^{\star}$ non tipici. La nuova specie se ne distingue per l'apice dell'edeago inciso, in visione ventrale, mentre è acutissimo e non inciso in tuberculicollis.

\section{Etimologia:}

La nuova specie evidentemente prende nome dalla Cina.

\section{Linoglossa (Axinocolya) chinensis PACE, 1998}

Linoglossa (Axinocolya) chinensis PACE, 1998: 202

1 ㅇ, China: Sichuan, Gongga Shan, Hailougou glacier park, env. camp II, 2650 m, 30.V.1997, sifted, leg. A. Pütz; 2 ㅇ ㅇ, China: N-Yunnan, Dali Bai Nat. Aut. Pref., Diancang Shan, 3 km W Dali, $25^{\circ} 41.1^{\prime} \mathrm{N} 100^{\circ} 07.8 \mathrm{E}, 29 . V I I I .2003$, leg. A. Smetana.

\section{Distribuzione:}

Specie già nota del Sichuan.

\section{Silusa sichuanensis PACE, 2004}

Silusa sichuanensis PACE, 2004: 70

1 ㅇ, China: Shaanxi, Daba Shan, creek vall SE pass $20 \mathrm{~km} \mathrm{NW}$ Zhenping, $1680 \mathrm{~m}, 31^{\circ} 59^{\prime} \mathrm{N}$ $109^{\circ} 22^{\prime}$ E, 11.VII.2001, leg. A. Smetana; 1 ㅇ, China: N-Yunnan, Zhongdian Co., pass $28 \mathrm{~km}$ ESE Zhongdian, $27^{\circ} 43.9^{\prime} \mathrm{N} 99^{\circ} 58.2^{\prime} \mathrm{E}, 3700-3750 \mathrm{~m}, 22 . \mathrm{VIII} .2003$, leg. A. Smetana; $3 \mathrm{o}^{\star} \mathrm{o}^{\star}$, China: N-Yunnan, Zhongdian Co., $36 \mathrm{~km}$ ESE Zhongdian, $27^{\circ} 40.9^{\prime} \mathrm{N} 100^{\circ} 01.5 \mathrm{E}, 3500-$ 3550 m, 23.VIII.2003, leg. A. Smetana. 


\section{Distribuzione:}

Specie finora nota del Sichuan.

\section{BOLITOCHARINi Thomson, 1859}

\section{Tibetusina n. gen.}

(Figg. 6 e 26-29)

\section{Diagnosi:}

A motivo della formula tarsale 4-4-5 e dei palpi labiali di tre articoli, il nuovo genere si pone nella tribù Bolitocharini. Per la forma della spermateca l'unico genere di questa tribù simile è Omologlusa PACE, 1998 pure della Cina, ma in questo genere la ligula è assai larga e corta, mentre nel nuovo genere è stretta e lunga. Il primo articolo dei palpi labiali del nuovo genere è lungo il doppio rispetto al secondo, mentre in Omologlusa il primo articolo dei palpi labiali è lungo quanto il secondo. Il terzo articolo dei palpi mascellari in Omologlusa è piriforme, largo il doppio della larghezza del secondo articolo, mentre nel nuovo genere il medesimo è persino più stretto del secondo.

\section{Descrizione:}

Labbro trasverso, arrotondato agli angoli anteriori, debolmente smarginato al margine anteriore. Mandibole prominenti; la mandibola destra con un piccolo dente o con una smarginatura solamente a metà del margine interno. Maxillae con il lobo esterno pubescente e membranoso all'apice; lobo interno ricurvo verso l'interno alla punta distale, con spine apicali lunghe, con setole sottili sulla porzione basale e membranosa. Palpi mascellari 4-articolati, moderatamente lunghi; terzo articolo più lungo e più stretto del secondo, non dilatato; quarto cortissimo e lesiniforme, fig. 29. Mento trapezoidale, debolmente smarginato all'orlo anteriore. Ligula stretta e intera, lunga quanto il primo articolo dei palpi labiali, fig. 28. Palpi labiali di tre articoli; articolo secondo molto più corto e più stretto del primo; terzo molto più stretto, ma più lungo del secondo. Antenne di undici articoli; i primi tre articoli più lunghi; articolo terzo più corto del secondo, antennomeri quarto a decimo fortemente trasversi, undicesimo lungo quasi quanto i due articoli precedenti riuniti. Capo molto prominente con tempio lievemente divergenti all'indietro, collo largo; occhi molto più corti delle tempie; parte inferiore delle tempie non marginate. Pronoto lungo quanto largo, più stretto delle elitre non sinuato davanti agli angoli posteriori esterni; margini laterali non ciliati. Elitre debolmente smarginate presso gli angoli posteriori esterni. Addome piuttosto parallelo, uroterghi liberi basali primo, secondo e terzo solcati trasversalmente alla base. Zampe corte; tarsi 4-4-5 articolati; primo protarsomero lungo quanto i due seguenti protarsomeri riuniti, primo mesotarsomero e primo metatarsomero appena più lungo del seguente e contiguo tarsomero. Processo mesosternale acuto. Mesocoxe contigue.

Spermateca descrivente una spira alla parte prossimale figg. 26-27.

\section{Typus generis:}

Tibetusina wolongensis n. sp.

\section{Etimologia:}

Il nome del nuovo genere significa «Piccola del Tibet» a motivo della taglia corporea ridotta, $1,7 \mathrm{~mm}$. 


\section{Tibetusina wolongensis $\mathrm{n} . \mathrm{sp}$.}

(Figg. 6 e 26-29)

\section{Materiale tipico:}

Holotypus + , China: W-Sichuan, Aba Tibetian Auton. Prefecture, Weizhou Co., Qionglai Shan, Wolong Valley, $20 \mathrm{~km}$ W Guanxian, 3104.65'N 10326.38'E, $1100 \mathrm{~m}, 14$.VII.1999, A. Pütz (SMTD).

Paratypi: 3 ㅇ , stessa provenienza.

\section{Descrizione:}

Lunghezza 1,75 mm. Corpo debolmente opaco e bruno, pronoto e pigidio giallo-bruni, antenne brune con i due antennomeri basali giallo-bruni, zampe gialle. Secondo antennomero più corto del primo, terzo più corto del secondo, antennomeri quarto a decimo fortemente trasversi. Reticolazione distinta sulla fascia longitudinale mediana del capo, assente sul resto della sua superficie. Reticolazione di pronoto e addome assente, quella delle elitre superficiale. Punteggiatura del capo fittissima e superficiale, assente sulla fascia longitudinale mediana. Granulosità del pronoto fittissima e superficiale, quella delle elitre fitta e poco ben visibile, quella dell'addome fitta e saliente, ma sugli uroterghi liberi quarto e quinto superficiale. Larga depressione mediana del pronoto. Spermateca figg. 26-27. Maschio sconosciuzto.

\section{Etimologia:}

La nuova specie prende nome dal luogo del suo ritrovamento, la Valle Wolong.

\section{Pseudatheta Cameron, 1920}

\section{Pseudatheta indica CAMERon, 1939}

Pseudatheta indica Cameron, 1939: 226

1 o , China: W-Sichuan, Ya'an Prefecture, Shimian Co., Xiaoxiang Ling, side valley ab. Nanya Cun, nr. Caluo, 11 km S Shimian, 1250 m, 7.VII.1999, leg. A. Pütz; 1 o $^{\star}$ e 1 ㅇ, China, Sichuan, Wennjian Distr., Guanxian Co, Qingcheng Shan, 56 km NW Chengdu, 3053.84'N 10332.80'E, 975 m, 18.VI.1999 m, 8 km W Taiping.VI.1997, leg. A. Pütz.

\section{Distribuzione:}

Specie già nota dell'India.

Nota:

Esemplari comparati con quelli della serie tipica da me esaminati.

\section{Pseudatheta ghoropanensis PACE, 1989}

Pseudatheta ghoropanensis PACE, 1989b: 536

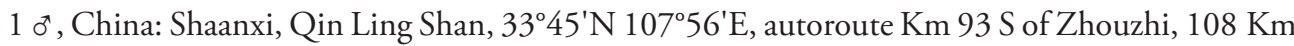
SW Xian, mountain forest, sifted, 1650 m, 1-2.IX.1995, leg. A. Pütz; 11 es., China: N-Yunnan, Zhongdian Co., $33 \mathrm{~km}$ ESE Zhongdian, 2741.5'N 10000.7'E, $3200 \mathrm{~m}$, 24.VIII.2003, leg. A. Smetana. 


\section{Distribuzione:}

Specie finora nota del Nepal.

Pseudatheta gonggaensis (PACE, 1998), comb. n.

Phymatura gonggaensis PACE, 1998: 206

$1 \mathrm{o}^{\top}$, China: N-Yunnan, Zhongdian Co., $36 \mathrm{~km} \mathrm{ESE} \mathrm{Zhongdian,} 27^{\circ} 40.9^{\prime} \mathrm{N} 100^{\circ} 01.5 \mathrm{E}$, 3500 m, 23.VIII.2003, leg. A. Smetana; 6 es., China: N-Yunnan, Zhongdian Co., 33 km ESE Zhongdian, $27^{\circ} 41.5^{\prime} \mathrm{N} 100^{\circ} 00.7^{\prime} \mathrm{E}, 3200 \mathrm{~m}, 24 . V I I I .2003$, leg. A. Smetana; $10^{\top}$, China: NYunnan, Zhongdian Co., pass $28 \mathrm{~km}$ ESE Zhongdian, $27^{\circ} 43.9^{\prime} \mathrm{N} 99^{\circ} 58.2^{\prime} \mathrm{E}, 3700-3750 \mathrm{~m}$, 22.VIII.2003, leg. A. Smetana.

\section{Distribuzione:}

Specie finora nota del Sichuan.

\section{Pseudatheta similis n. sp.}

(Figg. 7 e 30)

\section{Materiale tipico:}

Holotypus $0^{\star}$, China: Sichuan, Ganzi Prefecture, Daxue Shan, Gongga Shan, Hailougou glacier park, $102^{\circ} 04^{\prime} \mathrm{E} 29^{\circ} 36^{\prime} \mathrm{N}$, river valley, ca. $1 \mathrm{~km}$ above camp I, $2100 \mathrm{~m}, 28-31$.V.1997, leg. A. Pütz (SMTD).

Paratypi: $1 \sigma^{\star}$, stessa provenienza.

\section{Descrizione:}

Lunghezza 2,4 $\mathrm{mm}$. Corpo debolmente lucido e bruno, pronoto bruno-rossiccio, base delle elitre, base dell'addome e pigidio giallo-rossicci, antenne brune con i tre antennomeri basali giallo-rossicci, zampe giallo-rossicce. Secondo antennomero più lungo del primo, terzo più corto del secondo, antennomeri quarto a decimo fortemente trasversi. Reticolazione di capo ed elitre evidente, quella del pronoto forte, quella dell'addome trasversa e superficiale, tranne che sul quinto urotergo libero del $\sigma^{\star}$ su cui è forte. Punteggiatura del capo fine, fitta ed evidente, quella delle elitre fitta e superficiale. Granulosità del pronoto fine e saliente. Edeago fig. 30 .

\section{Comparazioni:}

L'edeago della nuova specie è simile a quello di $P$. indica CAmeron, 1939 dell'India, di cui ho esaminato $1 o^{*}$ e 1 o della serie tipica (Mus. London). L'apice della lama sternale dell'edeago, tuttavia, in visione laterale, è strettissimo in indica e assai largo nella nuova specie.

\section{Etimologia:}

Il nome della nuova specie sta ad indicare la somiglianza del suo edeago con quello di $P$. indica Cameron, 1939. 


\section{Pseudatheta chinensis n. sp.}

(Figg. 8 e 31-32)

\section{Materiale tipico:}

Holotypus $0^{\star}$, China: N.Yunnan, Lijiang, Naxi Aut. Co., E Yulongxue Shan, $30 \mathrm{~km} \mathrm{~N} \mathrm{Lijiang,}$ $27^{\circ} 09.0^{\prime} \mathrm{N} 100^{\circ} 14.9^{\prime} \mathrm{E}, 2800-2900 \mathrm{~m}, 13$.VIII.2003, leg. A. Smetana (SDEI).

\section{Descrizione:}

Lunghezza 2,8 mm. Corpo lucido e rossiccio, capo nero-bruno, elitre bruno-rossicce tranne la base rossiccia, fascia addominale mediana sugli uroterghi liberi primo, secondo e terzo e uroterghi liberi quarto e quinto bruni, antenne rossicce con i tre antennomeri basali giallo-rossicci, zampe giallo-rossicce. Secondo antennomero più lungo del primo, terzo più corto del secondo, antennomeri quarto a decimo fortemente trasversi. Reticolazione di capo e pronoto evidente, quella delle elitre forte. Granulosità di capo e pronoto evidente. Punteggiatura delle elitre netta e fitta. Edeago figg. 31-32.

\section{Comparazioni:}

L'habitus e l'edeago della nuova specie sono simili a quelli di $P$. indica CAmeron, 1939 dell'India, di cui ho esaminato $1 \sigma^{\star}$ e 1 o della serie tipica (Mus. London). La nuova specie se ne distingue per l'assenza di una lunga appendice ventrale dell'edeago, presente in indica, e per gli occhi lunghi quanto le tempie (occhi più corti delle tempie in indica).

\section{Etimologia:}

La nuova specie ovviamente prende nome dalla Cina.

Phymatura SAHLBERG, 1876

\section{Phymatura sichuanensis PACE, 2004}

\section{Phymatura sichuanensis PACE, 2004: 73}

12 es., China: Sichuan, Ganzi Prefecture, Daxue Shan, Gongga Shan, Hailougou glacier park, $102^{\circ} 04^{\prime} \mathrm{E} 29^{\circ} 36^{\prime} \mathrm{N}$, river valley, ca. $1 \mathrm{~km}$ above camp I, $2100 \mathrm{~m}, 28-31 . V .1997$, leg. A. Pütz; 4 es., China: Sichuan, Gongga Shan, Hailougou glacier park, above camp II, 3000 m, 30.V.1997, sifted, leg. A. Pütz.

\section{Distribuzione:}

Specie già nota del Sichuan.

\section{Phymatura sinica n. sp.}

(Figg. 9 e 33-35)

\section{Materiale tipico:}

Holotypus ơ ${ }^{\star}$, China: W-Sichuan, Ya'an Prefecture, Fulin Co., Daxiang Ling, Pass N Hanyuanjie, $51 \mathrm{~km}$ NNE Shimian, small stream, $2300 \mathrm{~m}, 10 . \mathrm{VII} .1999,29^{\circ} 39^{\prime} \mathrm{N} 102^{\circ} 37^{\prime} \mathrm{E}$, leg. A. Pütz (SMTD). 


\section{Descrizione:}

Lunghezza 3,6 mm. Corpo lucido e bruno, elitre bruno-rossicce, addome rossiccio con quarto urotergo libero bruno, antenne brune con i tre antennomeri basali rossicci, zampe giallo-rossicce. Secondo antennomero più corto del primo, terzo lungo quanto il secondo, quarto lungo quanto largo, antennomeri quinto a decimo fortemente trasversi. Occhi più lunghi delle tempie. Reticolazione di capo e pronoto forte, quella delle elitre evidente, quella dell'addome assente, tranne che sul terzo posteriore degli uroterghi liberi primo a quarto su cui è trasversa, quarto urotergo libero fortemente reticolato e quinto con maglie di reticolazione longitudinali. Punteggiatura del capo indistinta. Granulosità del pronoto fitta e molto saliente, quella delle elitre superficiale. Edeago figg. 33-34.

\section{Comparazioni:}

La nuova specie è distinta da $P$. sichuanensis PACE, 2004 per l'edeago meno profondamente arcuato al lato ventrale, con armatura genitale interna dell'edeago corta e tozza (lunga e slanciata in sichuanensis). Il pronoto lucido è poco trasverso nella nuova specie, quasi opaco e molto trasverso in sichuanensis.

\section{Etimologia:}

La nuova specie ha nome che significa «Abitatrice della Cina».

\section{Euryusa ERICHSON, 1837}

\section{Euryusa (Ectolabrus) submaculata MaRUyama \& HLAVÁč, 2001}

Euryusa (Ectolabrus) submaculata MaruYAMA \& Hlavíc, 2001: 180

$10^{\text {to }}$ e 2 우 우, China: N-Yunnan, Zhongdian Co., $33 \mathrm{~km}$ ESE Zhongdian, $27^{\circ} 41.5^{\prime} \mathrm{N} 100^{\circ} 00.7^{\prime} \mathrm{E}$, 3200 m, 24.VIII.2003, leg. A. Smetana.

\section{Distribuzione:}

Specie finora nota di Taiwan.

\section{Euryusa (Ectolabrus) minor MARUYAMA \& HLAVÁČ, 2001}

Euryusa (Ectolabrus) minor MaruYAMa \& HLaváč, 2001: 176

1 ․ China: Shaanxi, Daba Shan, creek vall SE pass $20 \mathrm{~km}$ NW Zhenping, 1700-1800 m, $31^{\circ} 44^{\prime} \mathrm{N} 109^{\circ} 35^{\prime} \mathrm{E}, 9 . \mathrm{VII} .2001$, leg. A. Smetana.

\section{Distribuzione:}

Specie finora nota del Giappone. 


\section{Bolitochara sinica n. sp.}

(Figg. 10 e 36-37)

\section{Materiale tipico:}

Holotypus $0^{\star}$, China: N-Yunnan, Zhongdian Co., $33 \mathrm{~km}$ ESE Zhongdian, $27^{\circ} 41.5^{\prime} \mathrm{N} 100^{\circ} 00.7^{\prime} \mathrm{E}$, 3200 m, 24.VIII.2003, leg. A. Smetana (SDEI).

\section{Descrizione:}

Lunghezza 3,5 mm. Corpo lucido e bruno, elitre e margine posteriore degli uroterghi liberi rossicci, antenne brune con i tre antennomeri basali rossicci, zampe rossicce con femori bruno-rossicci. Secondo antennomero più corto del primo, terzo più corto del secondo, quarto debolmente trasverso, antennomeri quinto a decimo trasversi. Reticolazione del corpo assente. Punteggiatura del capo superficiale e assente tra le antenne, quella del pronoto evanescente, quella delle elitre evidente e quella dell'addome forte. Pronoto con impressione mediana posteriore trasversa. Edeago figg. 36-37, sesto urotergo libero del ơ fig. 35.

\section{Comparazioni:}

La nuova specie è distinta da B. indica CAMEron, 1939, di cui ho esaminato l'holotypus ơ, per l'assenza di un profilo ventrale dell'edeago ad angolo accentuato come in indica e per l'armatura genitale interna dell'edeago più forte e lunga di quella di indica. Il quarto antennomero è debolmente trasverso nella nuova specie, lungo quanto largo in indica.

\section{Etimologia:}

La nuova specie ha nome che significa «Abitatrice della Cina».

\section{Eusteniamorphini Bernhauer \& Scheerpeltz, 1926}

Eusteniamorpha CAMERoN, 1920

\section{Eusteniamorpha jiajinensis n. sp.}

(Figg. 11 e 38-41)

\section{Materiale tipico:}

Holotypus o`, China: W-Sichuan, Ya’an Prefecture, Tianquan Co., Jiaajin Shan, valley above Labahe nr. Station, $57 \mathrm{~km}$ W Ya’an, $1800 \mathrm{~m}, 30^{\circ} 08^{\prime} 63^{\prime \prime} \mathrm{N} \mathrm{102} 25^{\prime} 18^{\prime \prime E}, 12$.VII.1999, light forest, leg. A. Pütz (SMTD).

\section{Descrizione:}

Lunghezza 1,45 mm. Corpo lucido e bruno-rossiccio, urotergo libero quarto bruno, antenne brune con i tre antennomeri basali giallo-rossicci, zampe giallo-rossicce. Secondo antennomero più corto del primo, terzo più corto del secondo, antennomeri quarto e quinto trasversi, sesto a decimo fortemente trasversi. Occhi lunghi quanto le tempie. Reticolazione del capo poligonale irregolare lievemente trasversa ed evidente, soprattutto sulla fronte. Resto del corpo senza reticolazione. Elitre perdute. Punteggiatura del capo fittissima, fine e superficiale, assente sulla fronte, Granulosità del pronoto finissima, poco saliente e fitta, quella dell'addome fitta e superficiale. Pronoto con solco mediano sulla metà distale e due solchi mediani ricurvi sulla metà prossimale, 
due fossette anteriori, fig. 40. Primo urotergo libero del $\sigma^{\star}$ carenato sulla linea mediana, fig. 41. Edeago figg. 38-39.

\section{Comparazioni:}

Per il sistema di solchi del pronoto, la nuova specie è chiaramente distinta dalle specie note della Cina, di cui una sola nota solo sulla + . L'edeago della nuova specie presenta un dentino ventrale come nell'edeago di E. ruiliensis PACE, 1998 dello Yunnan, ma la lunghezza dell'edeago è di $0,14 \mathrm{~mm}$ nella nuova specie, $0,30 \mathrm{~mm}$ in ruiliensis. Il pronoto, inoltre, è poco ristretto all'indietro nella nuova specie, fortemente ristretto in ruiliensis.

\section{Etimologia:}

La nuova specie prende nome dal Monte Jiajin.

\section{Falagrinni Mulsant \& Rey, 1873}

\section{Falagria LEACH, 1819}

\section{Falagria (Falagria) caesa ERICHSON, 1837}

Falagria caesa ERICHson, 1837: 295

Staphvlinus sulcatus PAYKull, 1789: 32 (nec Staphvlinus sulcatus 0. F. Muller, 1776: 97)

Falagria sulcata (PAYKull, 1789) auct.; PACE 1993: 71

1 ․ China: Shaanxi, Wei He, bank at bridge on autoroute to Xian Airport, $15 \mathrm{~km} \mathrm{~N}$ Xian, $400 \mathrm{~m}, 34^{\circ} 24^{\prime} \mathrm{N} 108^{\circ} 55^{\prime} \mathrm{E}, 22 . V I I I .1995$, leg. A. Pütz; 6 es., China: Beijing, International Airport, 400' $\mathrm{N} 116^{\circ} 35^{\prime} \mathrm{E}, 17 . \mathrm{V} .1997$, sifted, leg. A. Pütz; 16 es., China: Ying Kou Country, Liaoning Prov., 1987, leg. Li Jing Ke (coll. Klimaszewski).

\section{Distribuzione:}

Regioni paleartica, orientale e neartica.

\section{Falagria (Leptagria) shimianensis n. sp.}

(Figg. 12 e 42)

\section{Materiale tipico:}

Holotypus + , China: W-Sichuan, Ya’an Prefecture, Shimian Co., Xiaoxiang Ling Pass, betw. Shimian-Ganluo, $27 \mathrm{~km}$ SE Shimian, $2450 \mathrm{~m}$, 2902'75'N 102³1'48"E, 8.VII.1999, leg. A. Pütz (SMTD).

\section{Descrizione:}

Lunghezza 3,3 mm. Corpo lucido e bruno, elitre bruno-rossicce, antenne brune, zampe giallorossicce. Secondo antennomero lungo quanto il primo, terzo lungo quanto il secondo, quarto debolmente trasverso, antennomeri quinto a decimo trasversi. Reticolazione del corpo assente. Punteggiatura del capo fitta e molto superficiale, quella del pronoto fine ed evanescente. Granulosità dell'addome fitta sugli uroterghi liberi quarto a sesto, allineata trasversalmente sugli uroterghi liberi primo a terzo. Profondo solco mediano del pronoto. Spermateca fig. 42. 


\section{Comparazioni:}

La spermateca della nuova specie è simile a quella di F. vilis KraATz, 1859 dell'India, di cui ho esaminato l'holotypus ㅇ․ La spermateca di vilis è lunga $0,31 \mathrm{~mm}$, mentre quella della nuova specie $0,57 \mathrm{~mm}$. L'introflessione apicale del bulbo distale della spermateca di vilis è breve, lunga $0,034 \mathrm{~mm}$, mentre quella della nuova specie è di $0,1 \mathrm{~mm}$.

\section{Etimologia:}

La nuova specie prende nome dal toponimo Shimian.

\section{Falagria (Leptagria) hubeiensis n. sp.}

(Figg. 13 e 43)

\section{Materiale tipico:}

Holotypus o , China: W-Hubei, Daba Shan pass E of Mt. Da Shennongjia, 12 km NW Muyuping, $31^{\circ} 30^{\prime} \mathrm{N} 110^{\circ} 21^{\prime} \mathrm{E}, 19 . \mathrm{VII} .2001,2050 \mathrm{~m}$, leg. A. Smetana (SDEI).

\section{Descrizione:}

Lunghezza 2,5 mm. Corpo lucido e nero-bruno, elitre, antenne e margine posteriore dei tre uroterghi liberi basali bruni, zampe giallo-rossicce. Secondo antennomero lungo quanto il primo, terzo più corto del secondo, quarto debolmente trasverso, antennomeri quinto a decimo trasversi. Reticolazione del corpo assente, tranne che sulle elitre su cui è molto superficiale. Punteggiatura del capo superficiale. Granulosità del pronoto superficiale, quella delle elitre saliente alla base, fine sul resto delle elitre. Granulosità dell'addome evidente. Depressione trasversa tra le antenne. Profondo solco mediano del pronoto, confluente all'indietro in una fossetta trasversa. Spermateca fig. 43.

\section{Comparazioni:}

La spermateca della nuova specie è simile a quella di F. sichuanensis PACE, 1993 del Sichuan, ma il bulbo distale della spermateca è molto più stretto, $0,07 \mathrm{~mm}$, di quello di sichuanensis, $0,09 \mathrm{~mm}$, e l'introflessione apicale del bulbo distale della spermateca è poco profonda, $0,04 \mathrm{~mm}$, mentre in sichuanensis è profonda, $0,09 \mathrm{~mm}$. Il pronoto della nuova specie è trasverso, lungo quanto largo in sichuanensis.

\section{Etimologia:}

La nuova specie prende nome da Hubei.

\section{Falagria (Myrmecocephalus) dimidiata MoTsChulsky, 1858}

Falagria dimidiata MотsChulsкy, 1858: 260

Falagria (Stenagria) dimidiata; CAMERON 1939: 250

1 ㅇ , China: W-Sichuan, Yảan Prefecture, Shimian Co., Daxue Shan, W road btw. AnshunchangWanba, 20 km WSW Shimian, 1500 m, 9.VII.1999, leg. A. Pütz.

\section{Distribuzione:}

Specie diffusa nello Sri Lanka e in India. Nuova per la Cina. 
Falagria (Myrmecocephalus) pallipennis CAMERON, 1939

Falagria (Stenagria) pallipennis CAMERon, 1939: 253

4 es., China, Sichuan, Qingcheng Shan, 65 km NW Chengdu, 30 53'N 103³3'E, 800-1000 m, 18.V/3-4.VI.1999 m, 8 km WTaiping.VI.1997, leg. A. Pütz; 1 đo e 1 우, China, Sichuan, Wenjiang Distr., Guanxian Co., 56 km NW Chengou, Qingcheng Shan, 3053.84'N 10332.80'E, 13.VII.1999, leg. A. Pütz; $10^{\star}$, China: N-Yunnan, Dali Bai Nat. Aut. Pref., Diancang Shan, 3 km W Dali, $25^{\circ} 41.1^{\prime} \mathrm{N} 100^{\circ} 06.8 \mathrm{E}, 2750 \mathrm{~m}$, 1.IX.2003, leg. A. Smetana.

India, Thailandia e Cina.

\section{Distribuzione:}

Specie già nota di India, Thailandia e Cina.

Nota:

Serie tipica da me esaminata.

\section{Falagria (Myrmecocephalus) opacella CAMERON, 1939}

Falagria (Stenagria) opacella CAMEron, 1939: 253

1 ㅇ, China: N.Yunnan, Lijiang, Naxi Aut. Co., E Yulongxue Shan, 30 km N Lijiang, $27^{\circ} 09.0^{\prime} \mathrm{N}$ 100¹4.9'E, 2800-2900 m, 13.VIII.2003, leg. A. Smetana.

\section{Distribuzione:}

Specie già nota dell'India.

Nota:

Serie tipica da me esaminata.

\section{Falagria (Myrmecocephalus) magnifica n. sp.}

(Figg. 14 e 44-45)

\section{Materiale tipico:}

Holotypus đ`, China: Guangxi, Cenwanglaoshan, 1350 m, 31.VII.1999, leg. J. R. Fellow (IRSNB).

\section{Descrizione:}

Lunghezza $3 \mathrm{~mm}$. Corpo giallo-rossiccio, metà posteriore delle elitre, uroterghi liberi quarto e base del quinto bruno-rossicci, antenne giallo-rossicce con antennomero basale, decimo e undicesimo gialli, zampe rossicce con tarsi, femori anteriori e base dei femori mediani e posteriori giallorossicci. Secondo antennomero più lungo del primo, terzo più corto del secondo, quarto a decimo più lunghi che larghi. Reticolazione del corpo assente. Punteggiatura del capo fine ed evidente, assente sulla fascia longitudinale mediana, quella del pronoto fine e ben distinta. Un debole solco mediano posteriore confluente in una profonda fossetta mediana posteriore. Tre solchi basali degli uroterghi liberi fortemente punteggiati nel fondo. Edeago figg. 44-45. 


\section{Comparazioni:}

Il pronoto della nuova specie ha forma molto simile a quello di F xishanensis PACE, 1998 dello Xishan, ma è lucido (opaco in xishanensis) e privo di un profondo solco mediano. Di xishanensis non è noto il $o^{*}$.

\section{Etimologia:}

La nuova specie prende nome di «Magnifica» perché il suo corpo eccelle per eleganza e bellezza di colore.

\section{Anaulacaspis beijingensis (PACE, 1998)}

Melagria beijingensis PACE, 1998: 398

Anaulacaspis beijingensis; SMETANA, 2002: 422

86 es., China: Shaanxi, Wei He, bank at bridge on autoroute to Xian Airport, $15 \mathrm{~km}$ N Xian, $400 \mathrm{~m}, 34^{\circ} 24^{\prime} \mathrm{N} 108^{\circ} 55^{\prime} \mathrm{E}, 22$.VIII.1995, leg. A. Pütz.

\section{Distribuzione:}

Specie già nota di Beijing e Yingtaogou.

\section{Ringraziamenti}

Per avermi affidato in studio il materiale oggetto del presente lavoro rivolgo i miei più cordiali ringraziamenti ai colleghi Dr. Aleš Smetana di Ottawa, Michael Schülke di Berlino, Guillaume de Rougemont, Dr. J. Klimaszewski e Olaf Jäger del Senckenberg Museum für Tierkunde di Dresda. Per il prestito di tipi ringrazio il Dr. P. M. Hammond del Museo di Storia Naturale di Londra, il Dr. Didier Drugmand de l'Institut royal des Sciences naturelles de Belgique il Dr. Lothar Zerche del SDEI di Müncheberg, il Dr. A. Newton del Field Museum of Natural History di Chicago e il Dr. Manfred Uhlig del Museo Zoologico dell'Università Humboldt di Berlino.

\section{Bibliografia}

Bernhauer, M. \& Scheerpeltz, O. 1926: Staphylinidae 6: 499-988. Pars 82. - In: Junk, W. (ed.): Coleopterorum Catalogus, Berlin.

Cameron, M. 1920: New Species of Staphylinidae from Singapore. - Transactions of the Entomological Society of London 1920: 212-284.

Cameron, M. 1933: Staphylinidae (Col.) from Mount Kinabalu. - Journal of Federal Malay State Museum 17: 338-360.

Cameron, M. 1939: The Fauna of British India, including Ceylon and Burma. - Coleoptera, Staphylinidae 4: 410 pp., London.

Erichson, W. F. 1837: Die Käfer der Mark Brandenburg 1: 1-384, Berlin.

Fauvel, A. 1901: Liste des Staphylinidae du Japon central recueillis par M. le Dr. Harmand. - Bulletin du Muséum National d'Histoire Naturelle, Paris 1901: 62-66.

Heer, O. 1839: Fauna coleopterorum Helvetica. Pars I. fasc. 2. - Orelii, Fuesslini et Sociorum, Turici [Zurich]: pp. 145-360.

Kratz, G. 1857: Genera Aleocharinorum illustrata. - Linnaea Entomologica 11: 1-43.

KraAtz, G. 1859: Die Staphyliniden-Fauna von Ostindien, insbesondere der Insel Ceylan. - Archiv für Naturgeschichte 25: 1-196. 
LEACH, W. E. 1819: [New genera.] In Samouelle. - The entomologist's useful compendium, 496 pp, London.

Maruyama \& HlaváČ, 2001: Revision of the subgenus Ectolabrus of the genus Euryusa (Coleoptera: Staphylinidae: Aleocharinae). - Sociobiology 39 [2001-2002]: 167-185.

Motschulsky, V. De 1858: Enumération des nouvelles espèces de Coléoptères rapportées de ses voyages. - Bulletin de la Société impériale des Naturalistes de Moscou 3: 204-264.

Mulsant, E. \& Rey, C. 1871: Tribu des Brévipennes: Famille des Aléochariens: Septième branche: Myrmédoniaires. - Annales de la Société de Agriculture de Lyon 6: 33-738.

Mulsant, E. \& Rey, C. 1873: Description de divers Coléoptères Brévipennes nouveaux ou peu connus. - Opuscula Entomologica 15: 147-189.

Pace, R. 1989 a: Monografia del genere Leptusa KraAtZ. - Memorie del Museo civico di Storia naturale di Verona, II s. A: Biologica 8: 1-307.

PaCE, R. 1989b: Aleocharinae nepalesi del Museo di Ginevra Parte I (Coleoptera, Staphylinidae). - Revue suisse de Zoologie 96: 483-539.

PACE, R. 1993: Aleocharinae della Cina. - Bollettino del Museo civico di Storia naturale di Verona 17: 69-126.

PACE, R. 1998: Aleocharinae della Cina: Parte I. - Revue suisse de Zoologie 105 (1): 139-220.

PACE, R. 2004: Specie nuove o poco note di Homalotini, Silusini, Bolitocharini, Diestotini e Autaliini della Cina e della Thailandia (Coleoptera, Staphylinidae). - Revue suisse de Zoologie 111: 63-76.

SAHLBERG, J. 1876: Enumeratio Coleopterorum Brachelytrorum Fenniae I: Staphylinidae. - Acta Societatis pro Fauna Flora Fennica 1: 1-248.

Smetana, A. 2004: Aleocharinae, pp. 353-494. - In: Löвl, I. \& Smetana, A. (editors). - Catalogue of Palaearctic Coleoptera, Vol. 2. Stenstrup: Apollo Books: 942 pp.

Thomson, C. G. 1859: Skandinaviens Coleoptera, synoptikt bearbetade, I. - Berlingska Boktryckeriet, Lund: 290 pp.

\section{Indirizzo dell'autore:}

Roberto Pace

Via Vittorio Veneto, 13

37032 Monteforte d'Alpone (Verona), Italia

e-mail: pace.ent@tiscali.it

\section{Subject editor:}

Dr. L. Zerche 


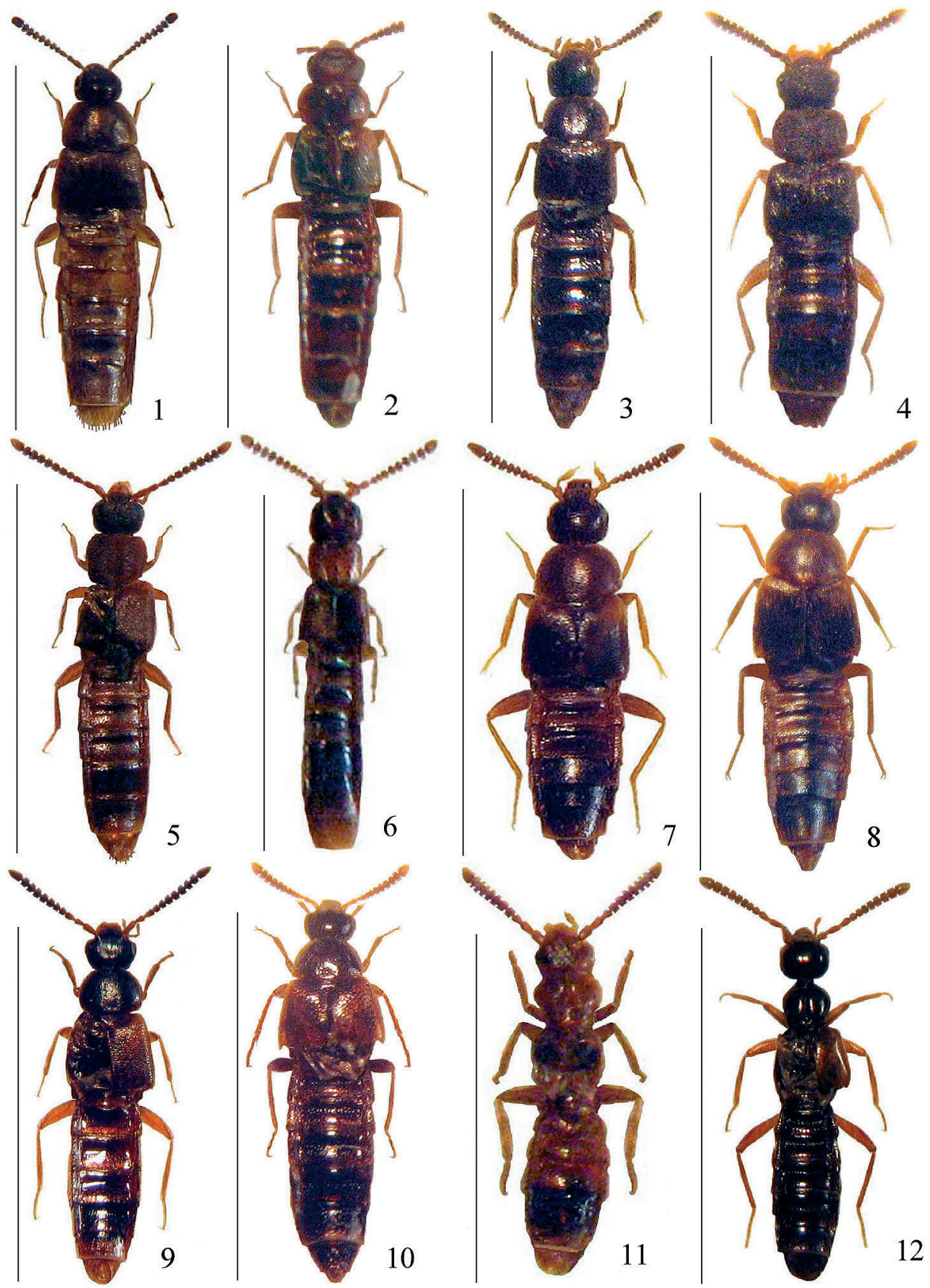

Figg. 1-12: Habitus. 1. Placusa shimianensis n. sp., scala $2,8 \mathrm{~mm}$; 2. Coenonica guanxiensis $\mathrm{n}$. sp., scala $3 \mathrm{~mm}$; 3. Coenonica confundibilis n. sp., scala $2,5 \mathrm{~mm}$; 4. Coenonica anteopaca n. sp., scala $2 \mathrm{~mm}$; 5. Stenomastax chinensis n. sp., scala $3 \mathrm{~mm}$; 6. Tibetusina wolongensis $\mathrm{n}$. gen., n. sp., scala 1,7 mm; 7. Pseudatheta similis n. sp., scala $2,4 \mathrm{~mm}$; 8. Pseudatheta chinensis n. sp., scala $2,8 \mathrm{~mm}$; 9. Phymatura sinica n. sp., scala $3,6 \mathrm{~mm} ; 10$. Bolitochara sinica n. sp., scala $3,5 \mathrm{~mm} ; 11$. Eusteniamorpha jiajinensis n. sp., scala 1,45 mm; 12. Falagria (Leptagria) shimianensis n. sp., scala $3,3 \mathrm{~mm}$. 
PACE, R.: Biodiversità delle Aleocharinae della Cina: Placusini, Homalotini ...

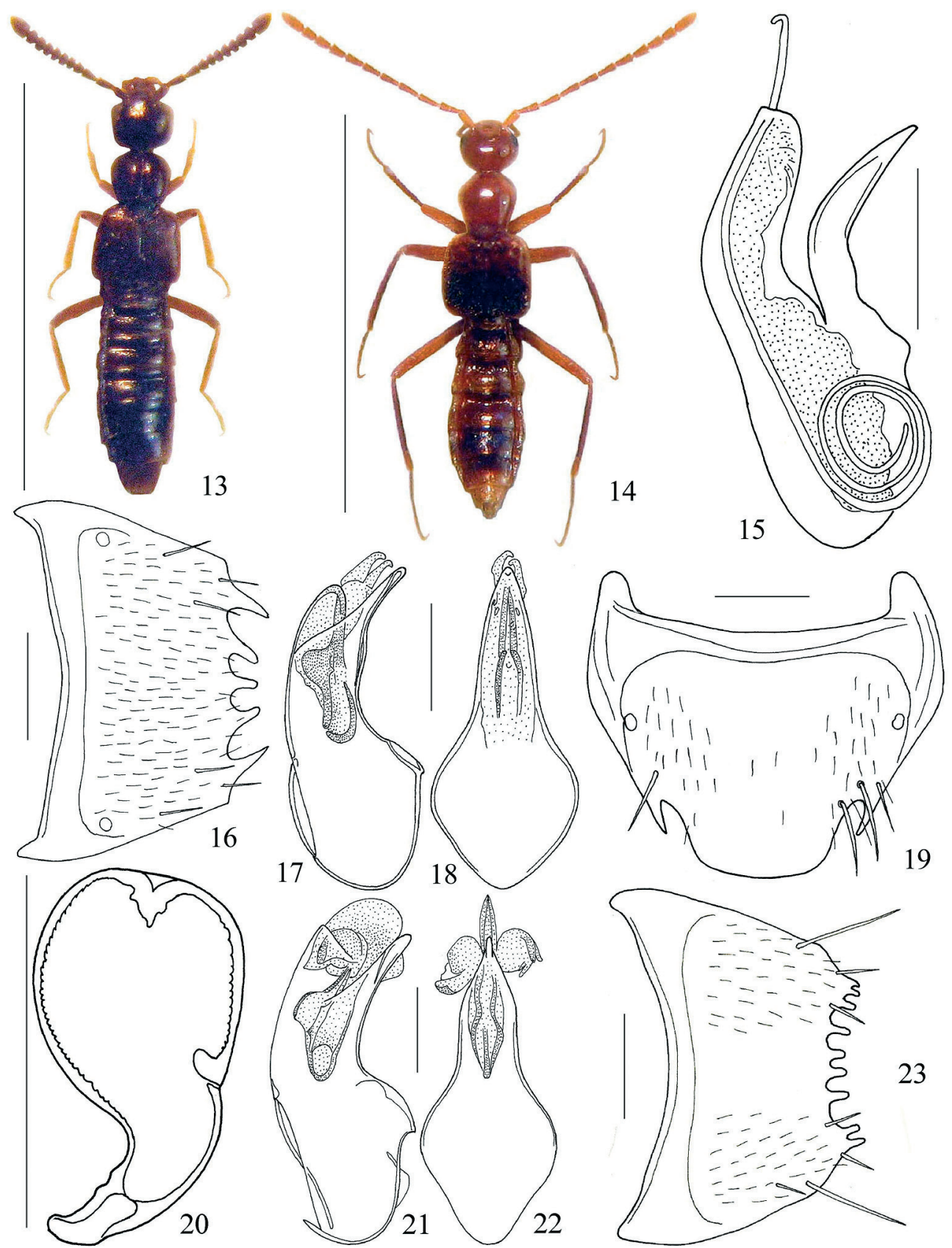

Figg. 13-23: Habitus, edeago in visione laterale e ventrale, sesto urotergo libero del ot e spermateca. 13. Falagria (Leptagria) hubeiensis n. sp., scala $2,5 \mathrm{~mm} ; 14$. Falagria (Myrmecocephalus) magnifica n. sp., scala 3 mm; 15-16. Placusa shimianensis n. sp.; 17-19. Coenonica guanxiensis $\mathrm{n}$. sp.; 20. Coenonica confundibilis n. sp.; 21-22. Coenonica anteopaca n. sp.; 23. Stenomastax chinensis n. sp. Altre scale 0,1 mm. 


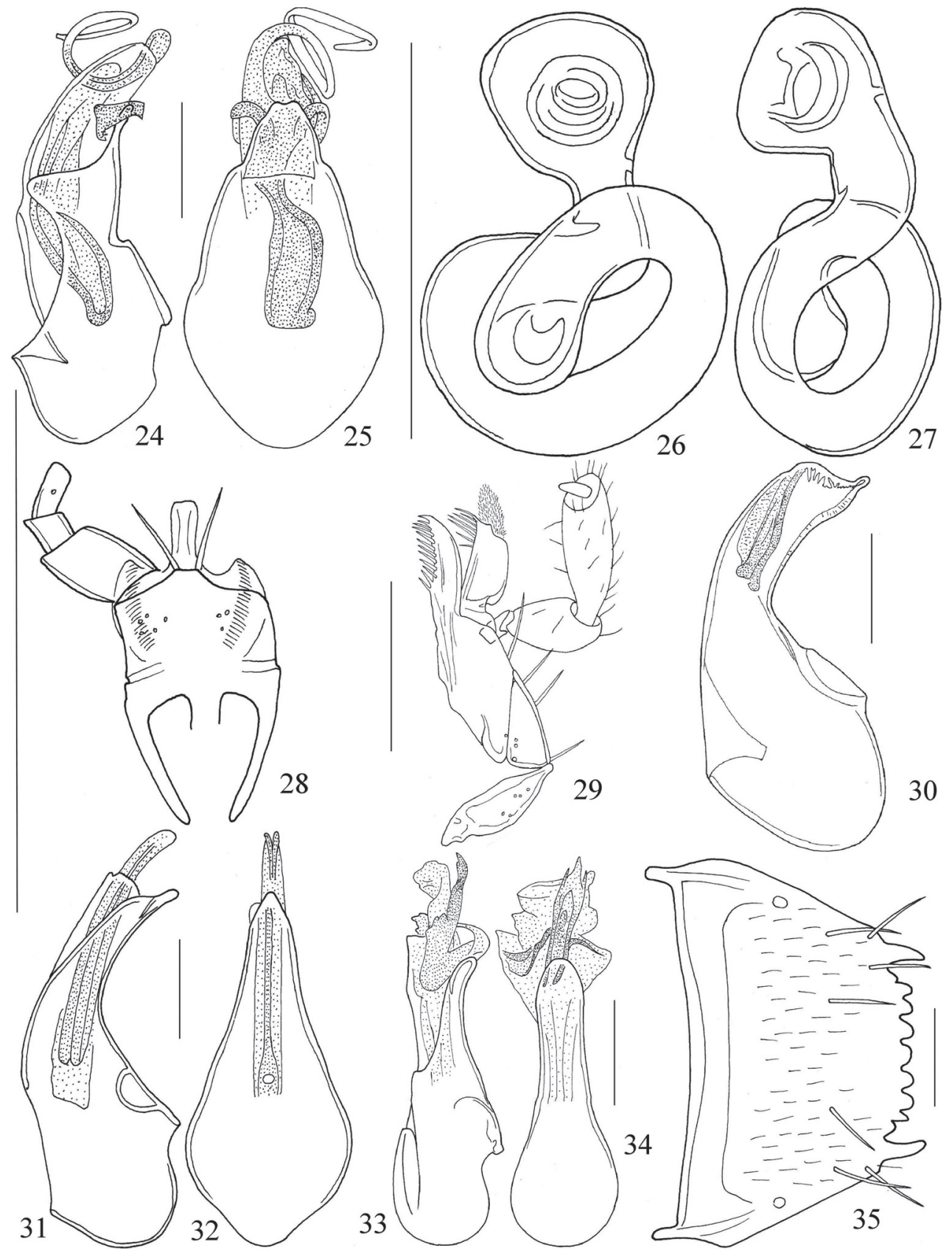

Figg. 24-35: Edeago in visione laterale e ventrale, spermateca, labio con palpo labiale, maxilla con palpo mascellare e sesto urotergo libero del $\sigma^{*}$. 24-25. Stenomastax chinensis $\mathrm{n}$. sp.; 26-29. Tibetusina wolongensis n. gen., n. sp.; 30. Pseudatheta similis n. sp.; 31-32. Pseudatheta chinensis n. sp.; 33-35. Phymatura sinica n. sp. Scale $0,1 \mathrm{~mm}$. 

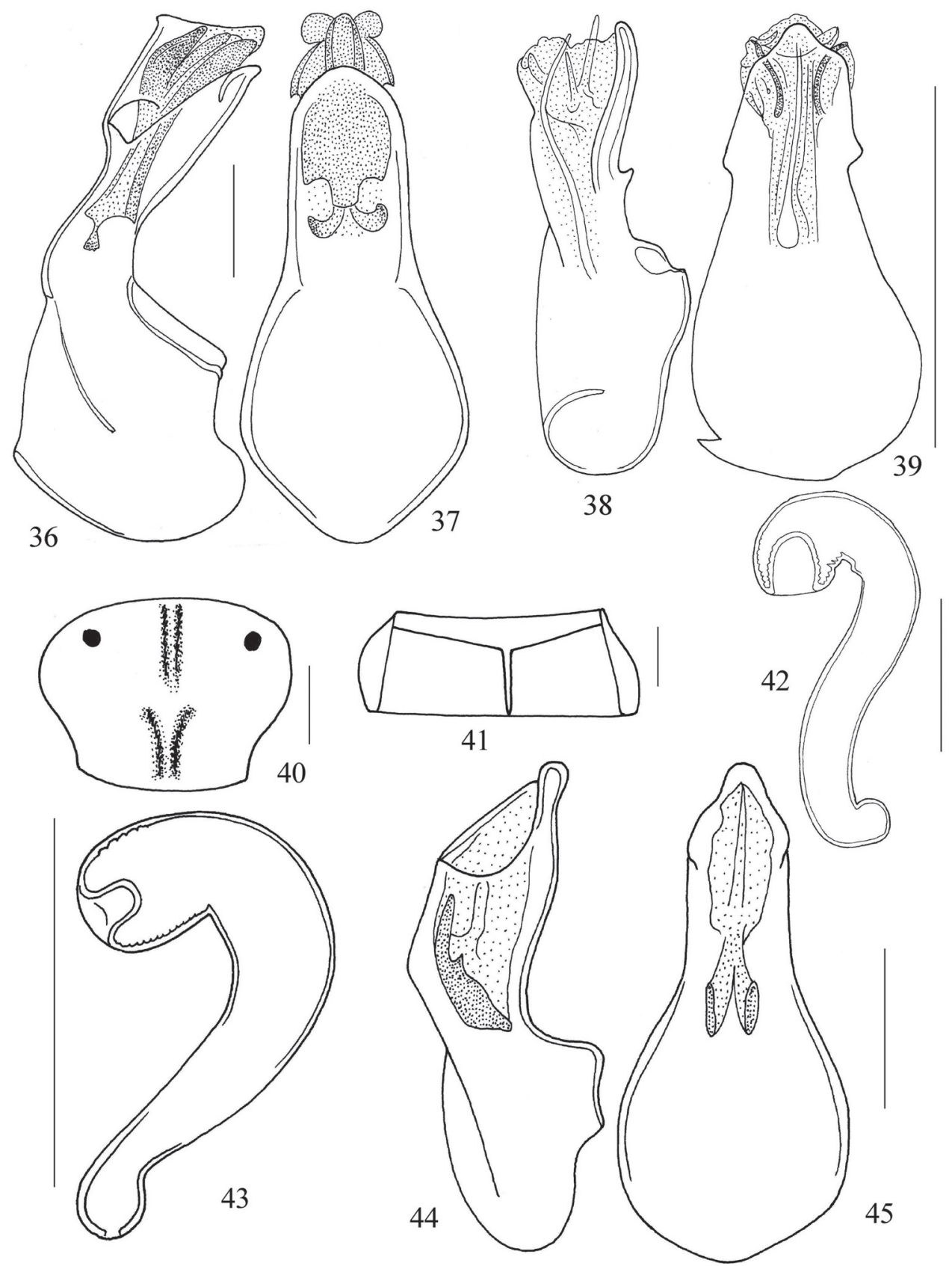

Figg. 36-45: Edeago in visione laterale e ventrale, pronoto, primo urotergo libero del o e spermateca. 36-37. Bolitochara sinica n. sp.; 38-41. Eusteniamorpha jiajinensis n. sp.; 42. Falagria (Leptagria) shimianensis n. sp.; 43. Falagria (Leptagria) hubeiensis n. sp.; 44-45. Falagria (Myrmecocephalus) magnifica n. sp. Scale $0,1 \mathrm{~mm}$. 